\title{
Presynaptic modulation of CA3 network activity
}

Kevin J. Staley, Mark Longacher, Jaideep S. Bains and Audrey Yee

Nature Neurosci. 1, 201-209 (1998).

Because of a printing error, Figs $4 c, 5 c$ and 6 were not properly reproduced. The corrected versions are printed below.

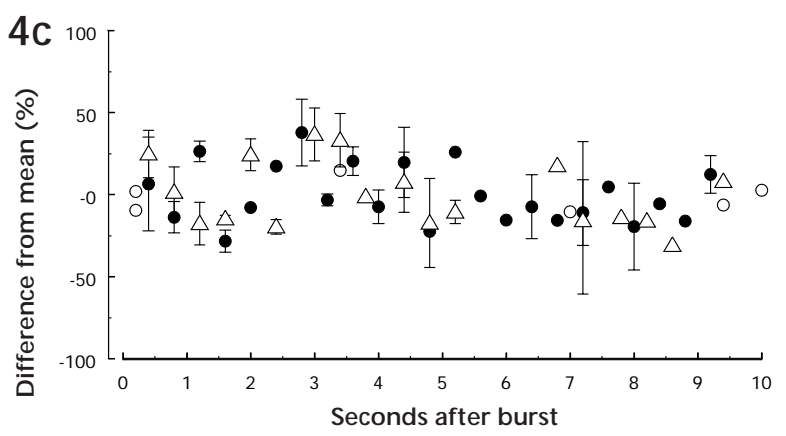

6a

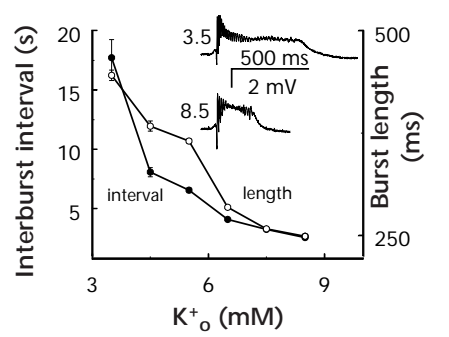

C
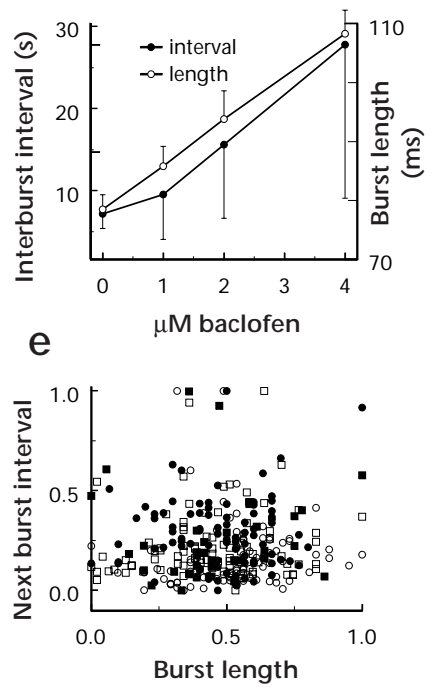

b

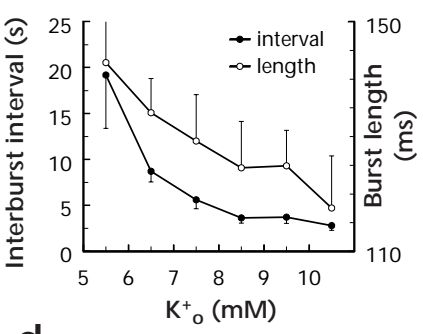

d
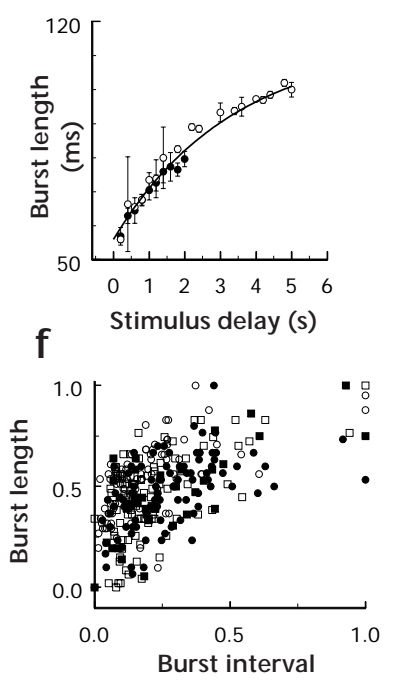

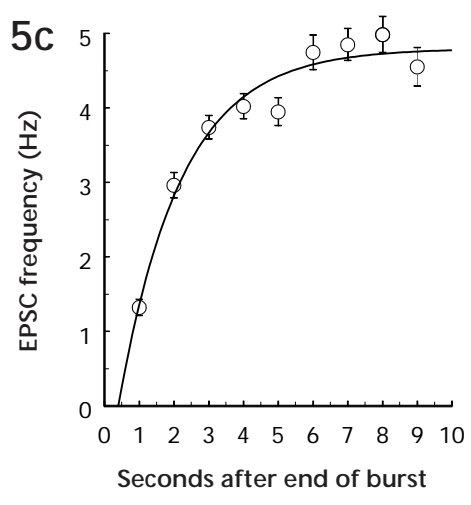

In addition, the penultimate sentence in the legend to Fig. $4 \mathrm{~b}$ was inadvertently omitted. The corrected legend should read:

Fig. 4..... (b) The experiment shown in (a) was repeated in a slice in which CA3 bursting was induced by tetanic stimulation of the CA3 pyramidal cell layer. The average interburst interval in this preparation was 13 seconds, which permitted longer delays between the end of a burst and glutamate application. As in (a), glutamate application at delays closer to the interburst interval triggered a burst more rapidly, but the initial response to glutamate was unchanged (inset). In this cell, bursts triggered calcium escape spikes. Glutamate was applied after every fourth burst in (b) and (c). 